A N N A LES

UNIVERSITATIS MARIAE CURIE-SKŁODOWSKA

LUBLIN - POLONIA

VOL. LXII, 2008

SECTIO A

$123-142$

SI DUC QUANG and TRAN VAN TAN

\title{
Uniqueness problem of meromorphic mappings with few targets
}

\begin{abstract}
In this paper, using techniques of value distribution theory, we give some uniqueness theorems for meromorphic mappings of $\mathbf{C}^{m}$ into $\mathbf{C} P^{n}$.
\end{abstract}

1. Introduction. Using the Second Main Theorem of Value Distribution Theory and Borel's lemma, R. Nevanlinna [11] proved that for two nonconstant meromorphic functions $f$ and $g$ on the complex plane $\mathbf{C}$, if they have the same inverse images for five distinct values, then $f \equiv g$, and that $g$ is a special type of linear fractional transformation of $f$ if they have the same inverse images, counted with multiplicities, for four distinct values.

In 1975, H. Fujimoto [5] generalized Nevanlinna's result to the case of meromorphic mappings of $\mathbf{C}$ into $\mathbf{C} P^{n}$. He showed that for two linearly nondegenerate meromorphic mappings $f$ and $g$ of $\mathbf{C}$ into $\mathbf{C} P^{n}$, if they have the same inverse images, counted with multiplicities for $(3 n+2)$ hyperplanes in $\mathbf{C} P^{n}$ located in general position, then $f \equiv g$, and there exists a projective linear transformation $L$ of $\mathbf{C} P^{n}$ to itself such that $g=L \cdot f$ if they have the same inverse images counted with multiplicities for $(3 n+1)$ hyperplanes in $\mathbf{C} P^{n}$ located in general position. Since that time, this problem has been studied intensively for the case of hyperplanes by H. Fujimoto ([7], [8]),

2000 Mathematics Subject Classification. 32H30, 32H04.

Key words and phrases. Meromorphic mappings, value distribution theory, uniqueness problem. 
W. Stoll [17], L. Smiley [14], S. Ji [9], M. Ru [13], Z. Ye [20], G. DethloffT. V. Tan ([2], [3], [4]), D. D. Thai-S. D. Quang [15] and others.

Let $f$ be a linearly nondegenerate meromorphic mapping of $\mathbf{C}^{m}$ into $\mathbf{C} P^{n}$. For each hyperplane $\mathrm{H}$, we denote by $\nu_{(f, H)}$ the map of $\mathbf{C}^{m}$ into $\mathbf{N}_{0}$ whose value $\nu_{(f, H)}(a)\left(a \in \mathbf{C}^{m}\right)$ is the intersection multiplicity of the image of $f$ and $H$ at $f(a)$.

Take $q$ hyperplanes $H_{1}, \ldots, H_{q}$ in $\mathbf{C} P^{n}$ located in general position with a) $\operatorname{dim}\left(f^{-1}\left(H_{i}\right) \cap f^{-1}\left(H_{j}\right)\right) \leq m-2$ for all $1 \leq i<j \leq q$.

For each positive integer (or $+\infty) M$, denote by $\mathcal{G}\left(\left\{H_{j}\right\}_{j=1}^{q}, f, M\right)$ the set of all linearly nondegenerate meromorphic mappings $g$ of $\mathbf{C}^{m}$ into $\mathbf{C} P^{n}$ such that

b) $\min \left\{\nu_{\left(g, H_{j}\right)}, M\right\}=\min \left\{\nu_{\left(f, H_{j}\right)}, M\right\}, j \in\{1, \ldots, q\}$ and

c) $g=f$ on $\bigcup_{j=1}^{q} f^{-1}\left(H_{j}\right)$.

In 1983, L. Smiley [14] showed that:

Theorem A. If $q \geq 3 n+2$ then $g_{1}=g_{2}$ for any $g_{1}, g_{2} \in \mathcal{G}\left(\left\{H_{j}\right\}_{j=1}^{q}, f, 1\right)$.

In 1998, H. Fujimoto [7] obtained the following theorem:

Theorem B. If $q \geq 3 n+1$ then $\mathcal{G}\left(\left\{H_{j}\right\}_{j=1}^{q}, f, 2\right)$ contains at most two mappings.

He also gave the open question: Does his result remain valid if the number of hyperplanes is replaced by a smaller one? In 2006, G. Dethloff and T. V. Tan [4] showed that the above result of Fujimoto remains valid if $q \geq 3 n-1$, $n \geq 7$. In this paper, by a different approach, we extend Theorem B to the case of

$$
q>\max \left\{\frac{7(n+1)}{4}, \frac{\sqrt{17 n^{2}+16 n}+3 n+4}{4}\right\} .
$$

In 1980, W. Stoll [19] obtained the following theorem:

Theorem C. Let $f_{1}, \ldots, f_{k}(k \geq 2)$ be linearly nondegenerate holomorphic mappings of $\mathbf{C}$ into $\mathbf{C} P^{n}$. Let $H_{1}, \ldots, H_{q}(q \geq(k+1) n+2)$ be hyperplanes in $\mathbf{C} P^{n}$ located in general position. Assume that

i) $f_{1}^{-1}\left(H_{j}\right)=\cdots=f_{k}^{-1}\left(H_{j}\right)$ for all $j \in\{1, \ldots, q\}$,

ii) $f_{1}^{-1}\left(H_{i}\right) \cap f_{1}^{-1}\left(H_{j}\right)=\emptyset$ for all $1 \leq i<j \leq q$ and

iii) $f_{1} \wedge \cdots \wedge f_{k}=0$ on $\bigcup_{j=1}^{q} f_{1}^{-1}\left(H_{j}\right)$.

Then $f_{1} \wedge \cdots \wedge f_{k} \equiv 0$.

In 2001, M. Ru [13] generalized the above result to the case of moving hyperplanes. In the last part of this paper, we extend Theorem $\mathrm{C}$ to the case of moving hypersurfaces.

Acknowledgements. The authors would like to thank Professors D. D. Thai, G. Dethloff, J. Nugochi for constant help and encouragement. 
2. Preliminaries. For $z=\left(z_{1}, \ldots, z_{m}\right) \in \mathbf{C}^{m}$, we set

$$
\|z\|=\left(\sum_{j=1}^{m}\left|z_{j}\right|^{2}\right)^{1 / 2}
$$

and define

$$
\begin{gathered}
B(r)=\left\{z \in \mathbf{C}^{m}:\|z\|<r\right\}, \quad S(r)=\left\{z \in \mathbf{C}^{m}:\|z\|=r\right\}, \\
d^{c}=\frac{\sqrt{-1}}{4 \pi}(\bar{\partial}-\partial), \mathcal{V}=\left(d d^{c}\|z\|^{2}\right)^{m-1}, \sigma=d^{c} \log \|z\|^{2} \wedge\left(d d^{c} \log \|z\|\right)^{m-1} .
\end{gathered}
$$

Let $F$ be a nonzero holomorphic function on $\mathbf{C}^{m}$. For a set $\alpha=\left(\alpha_{1}, \ldots, \alpha_{m}\right)$ of nonnegative integers, we set $|\alpha|=\alpha_{1}+\cdots+\alpha_{m}$ and $\mathcal{D}^{\alpha} F=\frac{\mathcal{D}^{|\alpha|} F}{\partial^{\alpha_{1}} z_{1} \ldots \partial^{\alpha_{m}} z_{m}}$. We define the map $\nu_{F}: \mathbf{C}^{m} \rightarrow \mathbf{N}_{0}$ by

$$
\nu_{F}(a)=\max \left\{p: \mathcal{D}^{\alpha} F(a)=0 \text { for all } \alpha \text { with }|\alpha|<p\right\} .
$$

Let $\varphi$ be a nonzero meromorphic function on $\mathbf{C}^{m}$. For each $a \in \mathbf{C}^{m}$, we choose nonzero holomorphic functions $F$ and $G$ on a neighborhood $U$ of $a$ such that $\varphi=\frac{F}{G}$ on $U$ and $\operatorname{dim}\left(F^{-1}(0) \cap G^{-1}(0)\right) \leq m-2$ and we define the map $\nu_{\varphi}: \mathbf{C}^{m} \longrightarrow \mathbf{N}_{0}$ by $\nu_{\varphi}(a)=\nu_{F}(a)$. Set

$$
\left|\nu_{\varphi}\right|=\overline{\left\{z: \nu_{\varphi}(z) \neq 0\right\}} \text {. }
$$

Let $k$ be positive integer or $+\infty$. Set $\nu_{\varphi}^{(k)}(z)=\min \left\{\nu_{\varphi}(z), k\right\}$, and

$$
N_{\varphi}^{(k)}(r):=\int_{1}^{r} \frac{n^{(k)}(t)}{t^{2 m-1}} d t \quad(1<r<+\infty)
$$

where

$$
n^{(k)}(t)=\int_{\left|\nu_{\varphi}\right| \cap B(t)} \nu_{\varphi}^{(k)} \cdot \mathcal{V} \text { for } m \geq 2
$$

and

$$
n^{(k)}(t)=\sum_{|z| \leq t} \nu_{\varphi}^{(k)}(z) \text { for } m=1
$$

We simply write $N_{\varphi}(r)$ for $N_{\varphi}^{(+\infty)}(r)$. We have the following Jensen's formula:

$$
N_{\varphi}(r)-N_{\frac{1}{\varphi}}(r)=\int_{S(r)} \log |\varphi| \sigma-\int_{S(1)} \log |\varphi| \sigma .
$$


Let $f$ be a meromorphic mapping of $\mathbf{C}^{m}$ into $\mathbf{C} P^{n}$. For arbitrary fixed homogeneous coordinates $\left(w_{0}: \cdots: w_{n}\right)$ of $\mathbf{C} P^{n}$, we take a reduced representation $f=\left(f_{0}: \cdots: f_{n}\right)$ which means that each $f_{i}$ is holomorphic function on $\mathbf{C}^{m}$ and $f(z)=\left(f_{0}(z): \cdots: f_{n}(z)\right)$ outside the analytic $I(f):=\left\{z: f_{0}(z)=\cdots=f_{n}(z)=0\right\}$ of codimension $\geq 2$. Set $\|f\|=\max \left\{\left|f_{0}\right|, \ldots,\left|f_{n}\right|\right\}$.

The characteristic function of $f$ is defined by

$$
T_{f}(r):=\int_{S(r)} \log \|f\| \sigma-\int_{S(1)} \log \|f\| \sigma, \quad 1<r<+\infty .
$$

For a meromorphic function $\varphi$ on $\mathbf{C}^{m}$, the characteristic function $T_{\varphi}(r)$ of $\varphi$ is defined as $\varphi$ is a meromorphic map of $\mathbf{C}^{m}$ into $\mathbf{C} P^{1}$. The proximity function $m(r, \varphi)$ is defined by

$$
m(r, \varphi)=\int_{S(r)} \log ^{+}|\varphi| \sigma,
$$

where $\log ^{+} x=\max \{\log x, 0\}$ for $x \geq 0$.

Then

$$
T_{\varphi}(r)=N_{\frac{1}{\varphi}}(r)+m(r, \varphi)+O(1) .
$$

We state the First and the Second Main Theorems of Value Distribution Theory:

Let $f$ be a nonconstant meromorphic mapping of $\mathbf{C}^{m}$ into $\mathbf{C} P^{n}$. We say that a meromorphic function $\varphi$ on $\mathbf{C}^{m}$ is "small" with respect to $f$ if $T_{\varphi}(r)=o\left(T_{f}(r)\right)$ as $r \rightarrow \infty$ (outside a set of finite Lebesgues measure). Denote by $\mathcal{R}_{f}$ the field of all "small" (with respect to $f$ ) meromorphic functions on $\mathbf{C}^{m}$.

Theorem D (First Main Theorem). Let $f$ be a nonconstant meromorphic mapping of $\mathbf{C}^{m}$ into $\mathbf{C} P^{n}$ and $Q$ be a homogeneous polynomial of degree $d$ in $\mathcal{R}_{f}\left[x_{0}, \ldots, x_{n}\right]$ such that $Q(f) \not \equiv 0$ then

$$
N_{Q(f)}(r) \leq d \cdot T_{f}(r)+o\left(T_{f}(r)\right) \text { for all } r>1 .
$$

For a hyperplane $H: a_{0} w_{0}+\cdots+a_{n} w_{n}=0$ in $\mathbf{C} P^{n}$ with $i m f \nsubseteq H$, we denote $(f, H):=a_{0} f_{0}+\cdots+a_{n} f_{n}$, where $\left(f_{0}: \cdots: f_{n}\right)$ again is a reduced representation of $f$.

As usual, by the notation "\| $\mathrm{P}$ " we mean the assertion $\mathrm{P}$ holds for all $r \in(1,+\infty)$ excluding a subset $E$ of $(1,+\infty)$ of finite Lebesgue measure.

Theorem E (Second Main Theorem). Let $f$ be a linearly nondegenerate meromorphic mapping of $\mathbf{C}^{m}$ into $\mathbf{C} P^{n}$ and $H_{1}, \ldots, H_{q}(q \geq n+1)$ hyperplanes in $\mathbf{C} P^{n}$ located in general position, then

$$
\|(q-n-1) T_{f}(r) \leq \sum_{j=1}^{q} N_{\left(f, H_{j}\right)}^{(n)}(r)+o\left(T_{f}(r)\right) .
$$


3. Uniqueness problem for hyperplanes. First of all, we give the following lemma, which is an extension of uniqueness theorem to the case of few hyperplanes.

Lemma 1. Let $f, g: \mathbf{C}^{m} \rightarrow \mathbf{C} P^{n}$ be two linearly nondegenerate meromorphic mappings with reduced representations $f=\left(f_{0}: \cdots: f_{n}\right), g=$ $\left(g_{0}: \cdots: g_{n}\right)$. Let $\left\{H_{i}\right\}_{i=1}^{q}$ be $q$ hyperplanes located in general position with $\operatorname{dim}\left(f^{-1}\left(H_{i}\right) \cap f^{-1}\left(H_{j}\right)\right) \leq m-2$ for all $1 \leq i<j \leq q$. Assume that

$$
q>\frac{\sqrt{17 n^{2}+16 n}+3 n+4}{4}
$$

and

(i) $\min \left\{\nu_{\left(f, H_{i}\right)}(z), n\right\}=\min \left\{\nu_{\left(g, H_{i}\right)}(z), n\right\}$, for all $i \in\{1, \ldots, q\}$,

(ii) Zero $\left(f_{j}\right) \cap f^{-1}\left(H_{i}\right)=\operatorname{Zero}\left(g_{j}\right) \cap f^{-1}\left(H_{i}\right)$, for all $1 \leq i \leq q, 0 \leq j \leq$ $n$,

(iii) $\mathcal{D}^{\alpha}\left(\frac{f_{k}}{f_{s}}\right)=\mathcal{D}^{\alpha}\left(\frac{g_{k}}{g_{s}}\right)$ on $\left(\bigcup_{i=1}^{q} f^{-1}\left(H_{i}\right)\right) \backslash\left(\right.$ Zero $\left.\left(f_{s}\right)\right)$, for all $|\alpha| \leq 1$, $0 \leq k \neq s \leq n$.

Then $f \equiv g$.

Proof. Assume that $f \not \equiv g$. We write $H_{i}: \sum_{j=0}^{n} a_{i j} \omega_{j}=0$.

For any fixed index $i,(1 \leq i \leq q)$, it is easy to see that there exists $j \in\{1, \ldots, q\} \backslash\{i\}$ (depending on $i$ ) such that

Set

$$
P_{i j}:=\frac{\left(f, H_{i}\right)}{\left(f, H_{j}\right)}-\frac{\left(g, H_{i}\right)}{\left(g, H_{j}\right)} \not \equiv 0 .
$$

$$
I:=I(f) \cup I(g) \cup \bigcup_{1 \leq k<s \leq q}\left\{z \in \mathbf{C}^{m}: \nu_{\left(f, H_{k}\right)}(z)>0 \text { and } \nu_{\left(f, H_{s}\right)}(z)>0\right\} .
$$

Then $I$ is an analytic subset of codimension $\geq 2$.

Case 1. $n \geq 2$.

Let $t$ be an arbitrary index in $\{1, \ldots, q\} \backslash\{i, j\}$. For any fixed point $z_{0} \notin I$ satisfying $\nu_{\left(f, H_{t}\right)}\left(z_{0}\right)>0$, there exists $l \in\{0, \ldots, n\}$ such that $f_{l}\left(z_{0}\right) g_{l}\left(z_{0}\right) \neq$ 0 . It follows that

$$
\begin{aligned}
\mathcal{D}^{\alpha} P_{i j}\left(z_{0}\right) & =\mathcal{D}^{\alpha}\left(\frac{\left(f, H_{i}\right)}{\left(f, H_{j}\right)}-\frac{\left(g, H_{i}\right)}{\left(g, H_{j}\right)}\right)\left(z_{0}\right) \\
& =\mathcal{D}^{\alpha}\left(\frac{\sum_{v=0}^{n} \frac{f_{v}}{f_{l}} a_{i v}}{\sum_{v=0}^{n} \frac{f_{v}}{f_{l}} a_{j v}}-\frac{\sum_{v=0}^{n} \frac{g_{v}}{g_{l}} a_{i v}}{\sum_{v=0}^{n} \frac{g_{v}}{g_{l}} a_{j v}}\right)\left(z_{0}\right)=0,
\end{aligned}
$$

for all $\alpha$ with $|\alpha|<2$. So

$$
\nu_{P_{i j}}\left(z_{0}\right) \geq 2 .
$$

For any fixed point $z_{1} \notin I$ satisfying $\nu_{\left(f, H_{i}\right)}\left(z_{1}\right)>0$, we have

$$
\nu_{P_{i j}}\left(z_{1}\right) \geq \min \left\{\nu_{\left(f, H_{i}\right)}\left(z_{1}\right), \nu_{\left(g, H_{i}\right)}\left(z_{1}\right)\right\} \geq \min \left\{\nu_{\left(f, H_{i}\right)}\left(z_{1}\right), n\right\} .
$$


From (3.1) and (3.2), we have

$$
\nu_{P_{i j}} \geq \min \left\{n, \nu_{\left(f, H_{i}\right)}\right\}+\sum_{t \in\{1, \ldots, q\} \backslash\{i, j\}} 2 \min \left\{1, \nu_{\left(f, H_{t}\right)}\right\},
$$

(outside an analytic subset of codimension two).

It yields that

$$
N_{P_{i j}}(r) \geq N_{\left(f, H_{i}\right)}^{(n)}(r)+\sum_{t \in\{1, \ldots, q\} \backslash\{i, j\}} 2 N_{\left(f, H_{t}\right)}^{(1)}(r)
$$

It is clear that

$$
N_{\frac{1}{P_{i j}}}(r) \leq N\left(r, \nu_{j}\right),
$$

where $\nu_{j}(z):=\max \left\{\nu_{\left(f, H_{j}\right)}(z), \nu_{\left(g, H_{j}\right)}(z)\right\}$.

We have

$$
\begin{aligned}
m\left(r, \frac{\left(f, H_{i}\right)}{\left(f, H_{j}\right)}\right) & =T_{\frac{\left(f, H_{i}\right)}{\left(f, H_{j}\right)}}(r)-N_{\left(f, H_{j}\right)}(r)+O(1) \\
& \leq T_{f}(r)-N_{\left(f, H_{j}\right)}(r)+O(1),
\end{aligned}
$$

and

$$
m\left(r, \frac{\left(g, H_{i}\right)}{\left(g, H_{j}\right)}\right) \leq T_{g}(r)-N_{\left(g, H_{j}\right)}(r)+O(1),
$$

This implies that

$$
\begin{aligned}
m\left(r, P_{i j}\right) & \leq m\left(r, \frac{\left(f, H_{i}\right)}{\left(f, H_{j}\right)}\right)+m\left(r, \frac{\left(g, H_{i}\right)}{\left(g, H_{j}\right)}\right)+O(1) \\
& =T_{f}(r)+T_{g}(r)-N_{\left(f, H_{j}\right)}(r)-N_{\left(g, H_{j}\right)}(r)+O(1) .
\end{aligned}
$$

Combining with (3.3) and (3.4) we get

$$
\begin{aligned}
N_{\left(f, H_{i}\right)}^{(n)}(r)+\sum_{t \in\{1, \ldots, q\} \backslash\{i, j\}} 2 N_{\left(f, H_{t}\right)}^{(1)}(r) \leq & N_{P_{i j}}(r) \leq T_{P_{i j}}(r)+O(1) \\
= & N_{\frac{1}{P_{i j}}}(r)+m\left(r, P_{i j}\right)+O(1) \\
\leq & T_{f}(r)+T_{g}(r)+N\left(r, \nu_{j}\right)-N_{\left(f, H_{j}\right)}(r) \\
& -N_{\left(g, H_{j}\right)}(r)+o\left(T_{f}(r)+T_{g}(r)\right) .
\end{aligned}
$$

This gives

$$
\begin{aligned}
N_{\left(f, H_{j}\right)}(r)+N_{\left(g, H_{j}\right)}(r)-N\left(r, \nu_{j}\right) & +N_{\left(f, H_{i}\right)}^{(n)}(r)+\sum_{t \in\{1, \ldots, q\} \backslash\{i, j\}} 2 N_{\left(f, H_{t}\right)}^{(1)}(r) \\
\leq & T_{f}(r)+T_{g}(r)+o\left(T_{f}(r)+T_{g}(r)\right) .
\end{aligned}
$$

On the other hand, since

$$
\nu_{j}(z)-\nu_{\left(f, H_{j}\right)}-\nu_{\left(g, H_{j}\right)}+\min \left\{n, \nu_{\left(f, H_{j}\right)}\right\} \leq 0
$$


(outside an analytic subset of codimension two), we have

$$
N\left(r, \nu_{j}\right)-N_{\left(f, H_{j}\right)}(r)-N_{\left(g, H_{j}\right)}(r)+N_{\left(f, H_{j}\right)}^{(n)}(r) \leq 0 .
$$

Hence

$$
\begin{aligned}
N_{\left(f, H_{i}\right)}^{(n)}(r)+N_{\left(f, H_{j}\right)}^{(n)}(r) & +\sum_{t \in\{1, \ldots, q\} \backslash\{i, j\}} 2 N_{\left(f, H_{t}\right)}^{(1)}(r) \\
& \leq T_{f}(r)+T_{g}(r)+o\left(T_{f}(r)+T_{g}(r)\right) .
\end{aligned}
$$

It implies that

$$
\begin{aligned}
N_{\left(f, H_{i}\right)}^{(n)}(r)+\frac{2}{n} & \sum_{t \in\{1, \ldots, q\} \backslash\{i\}} N_{\left(f, H_{t}\right)}^{(n)}(r) \\
& \leq T_{f}(r)+T_{g}(r)+o\left(T_{f}(r)+T_{g}(r)\right),
\end{aligned}
$$

(note that $n \geq 2$ ).

Taking summing-up of both sides of (3.5) over all $i \in\{1, \ldots, q\}$, we obtain

$$
\begin{aligned}
\left(1+\frac{2(q-1)}{n}\right) & \sum_{i=1}^{q} N_{\left(f, H_{i}\right)}^{(n)}(r) \\
& \leq q\left(T_{f}(r)+T_{g}(r)\right)+o\left(T_{f} f(r)+T_{g}(r)\right) .
\end{aligned}
$$

On the other hand, by Theorem $\mathrm{E}$ we have

$$
\|(q-n-1)\left(T_{f}(r)+T_{g}(r)\right) \leq 2 \sum_{i=1}^{q} N_{\left(f, H_{i}\right)}^{(n)}(r)+o\left(T_{f}(r)+T_{g}(r)\right) .
$$

From (3.6) and (3.7), letting $r \longrightarrow \infty$ we have

$$
1+\frac{2(q-1)}{n} \leq \frac{2 q}{q-n-1}
$$

This contradicts to

$$
q>\frac{\sqrt{17 n^{2}+16 n}+3 n+4}{4} .
$$

Thus $f \equiv g$.

Case 2. $n=1$. We have $q \geq 4$. If $\frac{\left(f, H_{1}\right)}{\left(f, H_{4}\right)} \equiv \frac{\left(g, H_{1}\right)}{\left(g, H_{4}\right)}$, then $f \equiv g$.

We now assume that

$$
P_{14}:=\frac{\left(f, H_{1}\right)}{\left(f, H_{4}\right)}-\frac{\left(g, H_{1}\right)}{\left(g, H_{4}\right)} \not \equiv 0 .
$$

Let $t$ be an arbitrary index in $\{1,2,3\}$. For any fixed point $z_{0} \notin I$ satisfying $\nu_{\left(f, H_{t}\right)}\left(z_{0}\right)>0$, there exists $l \in\{0,1\}$ such that $f_{l}\left(z_{0}\right) g_{l}\left(z_{0}\right) \neq 0$. It follows 
that

$$
\begin{aligned}
\mathcal{D}^{\alpha} P_{14}\left(z_{0}\right) & =\mathcal{D}^{\alpha}\left(\frac{\left(f, H_{1}\right)}{\left(f, H_{4}\right)}-\frac{\left(g, H_{1}\right)}{\left(g, H_{4}\right)}\right)\left(z_{0}\right) \\
& =\mathcal{D}^{\alpha}\left(\frac{a_{10} \frac{f_{0}}{f_{l}}+a_{11} \frac{f_{1}}{f_{l}}}{a_{40} \frac{f_{0}}{f_{l}}+a_{41} \frac{f_{1}}{f_{l}}}-\frac{a_{10} \frac{g_{0}}{g_{l}}+a_{11} \frac{g_{1}}{g_{l}}}{a_{40} \frac{g_{0}}{g_{l}}+a_{41} \frac{g_{1}}{g_{l}}}\right)\left(z_{0}\right)=0,
\end{aligned}
$$

for all $\alpha$ with $|\alpha|<2$. It implies that $\nu_{P_{14}}\left(z_{0}\right) \geq 2$. Hence, we have

$$
\nu_{P_{14}} \geq 2\left(\min \left\{1, \nu_{\left(f, H_{1}\right)}\right\}+\min \left\{1, \nu_{\left(f, H_{2}\right)}\right\}+\min \left\{1, \nu_{\left(f, H_{3}\right)}\right\}\right),
$$

(outside an analytic subset of codimension two). It implies that

$$
N_{P_{14}}(r) \geq 2\left(N_{\left(f, H_{1}\right)}^{(1)}(r)+N_{\left(f, H_{2}\right)}^{(1)}(r)+N_{\left(f, H_{3}\right)}^{(1)}(r)\right) .
$$

Let $z_{1}$ be an arbitrary pole of $P_{14}$ such that $z_{1} \notin I$. Then $z_{1}$ is a zero of $\left(f, H_{4}\right)$ and there exists $l \in\{0,1\}$ such that $f_{l}\left(z_{1}\right) g_{l}\left(z_{1}\right) \neq 0$. Then

$$
\begin{aligned}
\mathcal{D}^{\alpha} & \left(\left(a_{10} \frac{f_{0}}{f_{l}}+a_{11} \frac{f_{1}}{f_{l}}\right)\left(a_{40} \frac{g_{0}}{g_{l}}+a_{41} \frac{g_{1}}{g_{l}}\right)\right. \\
& \left.-\left(a_{40} \frac{f_{0}}{f_{l}}+a_{41} \frac{f_{1}}{f_{l}}\right)\left(a_{10} \frac{g_{0}}{g_{l}}+a_{11} \frac{g_{1}}{g_{l}}\right)\right)\left(z_{1}\right)=0,
\end{aligned}
$$

for all $\alpha$ with $|\alpha|<2$. This implies that

$$
\nu_{\left(\left(f, H_{1}\right)\left(g, H_{4}\right)-\left(f, H_{4}\right)\left(g, H_{1}\right)\right)}\left(z_{1}\right) \geq 2 .
$$

Then, we have

$$
\nu_{\frac{1}{P_{14}}}\left(z_{1}\right) \leq \nu_{\left(f, H_{4}\right)}\left(z_{1}\right)+\nu_{\left(g, H_{4}\right)}\left(z_{1}\right)-2 .
$$

Hence we see

$$
\nu_{\frac{1}{P_{14}}} \leq \nu_{\left(f, H_{4}\right)}+\nu_{\left(g, H_{4}\right)}-2 \min \left\{1, \nu_{\left(f, H_{4}\right)}\right\},
$$

(outside an analytic subset of codimension two). This implies that

$$
N_{\frac{1}{P_{14}}}(r) \leq N_{\left(f, H_{4}\right)}(r)+N_{\left(g, H_{4}\right)}(r)-2 N_{\left(f, H_{4}\right)}^{(1)}(r) .
$$


Combining with (3.8) we have

$$
\begin{aligned}
2\left(N_{\left(f, H_{1}\right)}^{(1)}(r)+\right. & \left.N_{\left(f, H_{2}\right)}^{(1)}(r)+N_{\left(f, H_{3}\right)}^{(1)}(r)\right) \leq N_{P_{14}}(r) \leq T_{P_{14}}(r)+O(1) \\
= & m\left(r, P_{14}\right)+N_{\frac{1}{P_{14}}}(r)+O(1) \\
\leq & m\left(r, \frac{\left(f, H_{1}\right)}{\left(f, H_{4}\right)}\right)+m\left(r, \frac{\left(g, H_{1}\right)}{\left(g, H_{4}\right)}\right) \\
& +N_{\left(f, H_{4}\right)}(r)+N_{\left(g, H_{4}\right)}(r)-2 N_{\left(f, H_{4}\right)}^{(1)}(r)+O(1) \\
= & T_{\frac{\left(f, H_{1}\right)}{\left(f, H_{4}\right)}}(r)+T_{\frac{\left(g, H_{1}\right)}{\left(g, H_{4}\right)}}(r)-2 N_{\left(f, H_{4}\right)}^{(1)}(r)+O(1) \\
\leq & T_{f}(r)+T_{g}(r)-2 N_{\left(f, H_{4}\right)}^{(1)}(r)+o\left(T_{f}(r)+T_{g}(r)\right) .
\end{aligned}
$$

It implies that

$$
\begin{aligned}
2\left(N_{\left(f, H_{1}\right)}^{(1)}(r)+N_{\left(f, H_{2}\right)}^{(1)}(r)\right. & \left.+N_{\left(f, H_{3}\right)}^{(1)}(r)+N_{\left(f, H_{4}\right)}^{(1)}(r)\right) \\
& \leq T_{f}(r)+T_{g}(r)+o\left(T_{f}(r)+T_{g}(r)\right) .
\end{aligned}
$$

On the other hand, by Theorem E, we also have

$$
\| 2 T_{f}(r) \leq N_{\left(f, H_{1}\right)}^{(1)}(r)+N_{\left(f, H_{2}\right)}^{(1)}(r)+N_{\left(f, H_{3}\right)}^{(1)}(r)+N_{\left(f, H_{4}\right)}^{(1)}(r)+o\left(T_{f}(r)\right)
$$

and

$$
\begin{aligned}
\| 2 T_{g}(r) & \leq N_{\left(g, H_{1}\right)}^{(1)}(r)+N_{\left(g, H_{2}\right)}^{(1)}(r)+N_{\left(g, H_{3}\right)}^{(1)}(r)+N_{\left(g, H_{4}\right)}^{(1)}(r)+o\left(T_{g}(r)\right) \\
& =N_{\left(f, H_{1}\right)}^{(1)}(r)+N_{\left(f, H_{2}\right)}^{(1)}(r)+N_{\left(f, H_{3}\right)}^{(1)}(r)+N_{\left(f, H_{4}\right)}^{(1)}(r)+o\left(T_{g}(r)\right)
\end{aligned}
$$

Hence, we have

$$
\| 2\left(T_{f}(r)+T_{g}(r)\right) \leq T_{f}(r)+T_{g}(r)+o\left(T_{f}(r)+T_{g}(r)\right) .
$$

Letting $r \longrightarrow \infty$, we have $2 \leq 1$. This is a contradiction, hence $f \equiv g$. We have completed the proof of Lemma 1.

Let $f$ be a linearly nondegenerate meromorphic mapping of $\mathbf{C}^{m}$ into $\mathbf{C} P^{n}$ with reduced representation $f=\left(f_{0}: \cdots: f_{n}\right)$. Let $d$ be a positive integer and let $H_{1}, \ldots, H_{q}$ be $q$ hyperplanes in $\mathbf{C} P^{n}$ located in general position with

$$
\operatorname{dim}\left\{z \in \mathbf{C}^{m}: \nu_{\left(f, H_{i}\right)}(z)>0 \text { and } \nu_{\left(f, H_{j}\right)}(z)>0\right\} \leq m-2
$$

$(1 \leq i<j \leq q)$.

Consider the set $\mathcal{F}\left(f,\left\{H_{j}\right\}_{j=1}^{q}, d\right)$ of all linearly nondegenerate meromorphic mappings $g: \mathbf{C}^{m} \rightarrow \mathbf{C} P^{n}$ with reduced representation $g=\left(g_{0}: \cdots\right.$ : $\left.g_{n}\right)$ satisfying the conditions:

(a) $\min \left(\nu_{\left(f, H_{i}\right)}, d\right)=\min \left(\nu_{\left(g, H_{i}\right)}, d\right)(1 \leq i \leq q)$,

(b) Zero $\left(f_{j}\right) \cap f^{-1}\left(H_{i}\right)=\operatorname{Zero}\left(g_{j}\right) \cap f^{-1}\left(H_{i}\right)$, for all $1 \leq i \leq q, 0 \leq j \leq$ $n$, 
(c) $\mathcal{D}^{\alpha}\left(\frac{f_{k}}{f_{s}}\right)=\mathcal{D}^{\alpha}\left(\frac{g_{k}}{g_{s}}\right)$ on $\left(\bigcup_{i=1}^{q} f^{-1}\left(H_{i}\right)\right) \backslash\left(Z \operatorname{ero}\left(f_{s}\right)\right)$, for all $|\alpha|<d$, $0 \leq k \neq s \leq n$.

Take $M+1$ maps $f^{0}, \ldots, f^{M} \in \mathcal{F}\left(f,\left\{H_{j}\right\}_{j=1}^{q}, d\right)$ with reduced representations

$$
f^{k}:=\left(f_{0}^{k}: \cdots: f_{n}^{k}\right)
$$

and set $T(r):=\sum_{k=0}^{M} T_{f^{k}}(r)$. For each $c=\left(c_{0}, \ldots, c_{n}\right) \in \mathbf{C}^{n+1} \backslash\{0\}$ we put

$$
\left(f^{k}, c\right):=\sum_{i=0}^{n} c_{i} f_{i}^{k} \quad(0 \leq k \leq M) .
$$

Denote by $\mathcal{C}$ the set of all $c \in \mathbf{C}^{n+1} \backslash\{0\}$ such that

$$
\operatorname{dim}\left\{z \in \mathbf{C}^{m}:\left(f^{k}, H_{j}\right)(z)=\left(f^{k}, c\right)(z)=0\right\} \leq m-2
$$

$(1 \leq j \leq q, 0 \leq k \leq M)$.

Lemma A ([9], Lemma 5.1). $\mathcal{C}$ is dense in $\mathbf{C}^{n+1}$.

Lemma B $([7])$. For each $c \in \mathcal{C}$, we put $F_{c}^{j k}=\frac{\left(f^{k}, H_{j}\right)}{\left(f^{k}, c\right)}$. Then $T_{F_{c}^{j k}}(r) \leq$ $T_{f^{k}}(r)+o(T(r))$.

Definition 1. Let $F_{0}, \ldots, F_{M}$ be meromorphic functions on $\mathbf{C}^{m}$, where $M \geq 1$. Take a set $\alpha:=\left(\alpha^{0}, \ldots, \alpha^{M-1}\right)$ whose components $\alpha^{k}$ are composed of $n$ nonnegative integers, and set $|\alpha|=\left|\alpha^{0}\right|+\cdots+\left|\alpha^{M-1}\right|$. We define Cartan's auxiliary function by

$$
\begin{aligned}
\Phi^{\alpha}\left(F_{0}, \ldots, F_{M}\right):=F_{0} & \cdot F_{1} \cdots F_{M} \\
\times & \left|\begin{array}{cccc}
1 & 1 & \cdots & 1 \\
\mathcal{D}^{\alpha^{0}}\left(\frac{1}{F_{0}}\right) & \mathcal{D}^{\alpha^{0}}\left(\frac{1}{F_{1}}\right) & \cdots & \mathcal{D}^{\alpha^{0}}\left(\frac{1}{F_{M}}\right) \\
\vdots & \vdots & \vdots & \vdots \\
\mathcal{D}^{\alpha^{M-1}}\left(\frac{1}{F_{0}}\right) & \mathcal{D}^{\alpha^{M-1}}\left(\frac{1}{F_{1}}\right) & \cdots & \mathcal{D}^{\alpha^{M-1}}\left(\frac{1}{F_{M}}\right)
\end{array}\right| .
\end{aligned}
$$

Lemma C ([7], Proposition 3.4). If $\Phi^{\alpha}(F, G, H)=0$ and $\Phi^{\alpha}\left(\frac{1}{F}, \frac{1}{G}, \frac{1}{H}\right)=0$ for all $\alpha$ with $|\alpha| \leq 1$, then one of the following conditions holds:

i) $F=G$ or $G=H$ or $H=F$.

ii) $\frac{F}{G}, \frac{G}{H}$ and $\frac{H}{F}$ are all constant.

Lemma 2. Assume that there exists $\Phi^{\alpha}:=\Phi^{\alpha}\left(F_{c}^{j_{0} 0}, \ldots, F_{c}^{j_{0} M}\right) \not \equiv 0$ for some $c \in \mathcal{C},|\alpha| \leq \frac{M(M-1)}{2}, d \geq|\alpha|$. Then, for each $0 \leq i \leq M$, the following holds:

$$
\| N_{\left(f^{i}, H_{j_{0}}\right)}^{(d-|\alpha|)}(r)+M d \sum_{j \neq j_{0}} N_{\left(f^{i}, H_{j}\right)}^{(1)}(r) \leq N_{\Phi^{\alpha}}(r) \leq T(r)+o(T(r)) .
$$


Proof. Denote by $\mathbf{P}$ the set of all $\beta$ with $|\beta| \leq \frac{M(M-1)}{2}, d \geq|\beta|$ such that $\Phi^{\beta}=\Phi^{\beta}\left(F_{c}^{j_{0} 0}, \ldots, F_{c}^{j_{0} M}\right) \not \equiv 0$ for some $c \in \mathcal{C}$. Let $\alpha$ be the minimal multi-index in $\mathbf{P}$ (in the lexicographic order). Set

$$
\begin{aligned}
I:=\bigcup_{t=0}^{M} I\left(f^{t}\right) & \cup \bigcup_{1 \leq t<j \leq q}\left(\left(f, H_{t}\right)^{-1}\{0\} \cap\left(f, H_{j}\right)^{-1}\{0\}\right) \\
& \cup \bigcup_{t=1}^{q}\left(\left(f, H_{t}\right)^{-1}\{0\} \cap(f, c)^{-1}\{0\}\right) .
\end{aligned}
$$

Then $I$ is an analytic subset of codimension $\geq 2$.

Assume that $a$ is a zero of some $\left(f^{i}, H_{j}\right), j \neq j_{0}$ such that $a \notin I$. Let $\Gamma$ be an irreducible component of the zero-divisor of the function $\left(f^{i}, H_{j}\right)$ which contains $a$. We take a holomorphic function $h$ on $C^{m}$ satisfying: $\left.\nu_{h}\right|_{\Gamma}=1$ and $\nu_{\left.h\right|_{\left(C^{n} \backslash \Gamma\right)}}=0$.

By the condition (c), we have that $\varphi_{i}:=\left(\frac{1}{h^{d} F^{j_{0}}}-\frac{1}{h^{d} F_{0}^{j_{0} M}}\right)$ is a holomorphic function on a neighborhood $U$ of $a$ for all $i \in\{0, \ldots, M-1\}$. Since $\alpha:=\min \mathbf{P}$, we have

$$
\Phi^{\alpha}:=h^{M d} F^{j_{0} 0} \cdots F^{j_{0} M} \times\left|\begin{array}{ccc}
\mathcal{D}^{\alpha^{0}} \varphi_{0} & \cdots & \mathcal{D}^{\alpha^{0}} \varphi_{M-1} \\
\vdots & \vdots & \vdots \\
\mathcal{D}^{\alpha^{M-1}} \varphi_{0} & \cdots & \mathcal{D}^{\alpha^{M-1}} \varphi_{M-1}
\end{array}\right|
$$

It implies that

$$
\nu_{\Phi^{\alpha}}(a) \geq M d
$$

Assume that $b$ is a zero of $\left(f^{i}, H_{j_{0}}\right)$ such that $b \notin I$. If $\nu_{\left(f^{i}, H_{j_{0}}\right)}(b) \geq d$, we write

$$
\begin{aligned}
\Phi^{\alpha}=\sum_{\sigma \in S_{M+1}} \operatorname{sign}(\sigma) & F^{j_{0} 0} \cdots F^{j_{0} M} \\
& \times \mathcal{D}^{\alpha^{0}}\left(\frac{1}{F^{j_{0}(\sigma(2)-1)}}\right) \cdots D^{\alpha^{M-1}}\left(\frac{1}{F^{j_{0}(\sigma(M+1)-1)}}\right) .
\end{aligned}
$$

Then

$$
\nu_{\Phi^{\alpha}}(b) \geq d-|\alpha|
$$

If $\nu_{\left(f^{i}, H_{j_{0}}\right)}(b)<d$, then $\nu_{\left(f^{0}, H_{j_{0}}\right)}(b)=\cdots=\nu_{\left(f^{M}, H_{j_{0}}\right)}(b)<d$. There exists a holomorphic function $h$ on an open neighborhood $U$ of $b$ such that $\nu_{h}=\nu_{\left(f^{i}, H_{j_{0}}\right)_{\left.\right|_{U}}}$. 
We write

$$
\begin{aligned}
& \Phi^{\alpha}=h^{-M} F_{c}^{j_{0} 0} \cdots F_{c}^{j_{0} M} \\
& \times\left|\begin{array}{ccc}
\left(\mathcal{D}^{\alpha^{0}}\left(\frac{h}{F_{c}^{j_{0}} 0}\right)-D^{\alpha^{0}}\left(\frac{h}{F_{c}^{j_{0} M}}\right)\right) & \cdots & \left(\mathcal{D}^{\alpha^{0}}\left(\frac{h}{F_{c}^{j_{0}(M-1)}}\right)-D^{\alpha^{0}}\left(\frac{h}{F_{c}^{j_{0} M}}\right)\right) \\
\vdots & \vdots & \vdots \\
\left(\mathcal{D}^{\alpha^{M-1}}\left(\frac{h}{F_{c}^{j_{0} 0}}\right)-D^{\alpha^{M-1}}\left(\frac{h}{F_{c}^{j_{0} M}}\right)\right) & \cdots & \left(\mathcal{D}^{\alpha^{M-1}}\left(\frac{h}{F_{c}^{j_{0}(M-1)}}\right)-D^{\alpha^{M-1}}\left(\frac{h}{F_{c}^{j_{0} M}}\right)\right)
\end{array}\right| .
\end{aligned}
$$

Then

$$
\nu_{\Phi^{\alpha}}(b) \geq \nu_{\left(f^{i}, H_{j_{0}}\right)}(b) .
$$

From (3.9), (3.10) and (3.11), we have

$$
\min \left\{d-|\alpha|, \nu_{\left(f^{i}, H_{j_{0}}\right)}\right\}+\underset{j \in\{1, \ldots, q\} \backslash\left\{j_{0}\right\}}{M d} \sum_{\min }\left\{1, \nu_{\left(f^{i}, H_{j}\right)}\right\} \leq \nu_{\Phi^{\alpha}},
$$

(outside an analytic subset of codimension two). It immediately follows the first inequality in the lemma.

It is easy to see that a pole of $\Phi^{\alpha}$ is a zero or a pole of some $F_{c}^{j_{0} k}$. By $(3.9),(3.10)$ and (3.11) we have that $\Phi^{\alpha}$ is holomorphic at all zeros of $F_{c}^{j_{0} i}$, $(0 \leq i \leq M)$. Then

$$
N_{\frac{1}{\Phi^{\alpha}}}(r) \leq \sum_{i=0}^{M} N_{\frac{1}{F_{c}^{j} 0^{i}}}(r) .
$$

On the other hand, it is easy to see that

$$
\begin{aligned}
m\left(r, \Phi^{\alpha}\right) & \leq \sum_{i=0}^{M} m\left(r, F_{c}^{j_{0} i}\right)+O\left(\sum m\left(r, \frac{\mathcal{D}^{\alpha^{i}}\left(\varphi_{c}^{j_{0} k}\right)}{\varphi_{c}^{j_{0} k}}\right)\right)+O(1) \\
& \leq \sum_{i=0}^{M} m\left(r, F_{c}^{j_{0} i}\right)+o(T(r)),
\end{aligned}
$$

where $\varphi_{c}^{j_{0} k}=1 / F_{c}^{j_{0} k}$. Hence, we have

$$
\begin{aligned}
N_{\Phi^{\alpha}}(r) & \leq T_{\Phi^{\alpha}}(r)+O(1) \leq m\left(r, \Phi^{\alpha}\right)+N_{\frac{1}{\Phi^{\alpha}}}(r)+O(1) \\
& \leq \sum_{i=0}^{M}\left(N_{\frac{1}{F_{c}^{j} 0^{i}}}(r)+m\left(r, F_{c}^{j_{0} i}\right)\right)+o(T(r)) \\
& =\sum_{i=0}^{M} T_{F_{c}^{j_{0} i}}(r)+o(T(r)) \leq T(r)+o(T(r)) .
\end{aligned}
$$

Theorem 1. If

$$
q>\max \left\{\frac{7(n+1)}{4}, \frac{\sqrt{17 n^{2}+16 n}+3 n+4}{4}\right\}
$$


then $\mathcal{F}\left(f,\left\{H_{i}\right\}_{i=1}^{q}, 2\right)$ contains at most two mappings.

Proof. If $n=1$, by Lemma 1 we have $\sharp \mathcal{F}\left(f,\left\{H_{i}\right\}_{i=1}^{q}, 1\right)=1$.

We prove the theorem for the case of $n \geq 2$. Assume that there exist three distinct mappings $f^{0}, f^{1}, f^{2} \in \mathcal{F}\left(f,\left\{H_{i}\right\}_{i=1}^{q}, 2\right)$.

Denote by $\mathcal{Q}$ the set of all indices $j \in\{1,2, \ldots, q\}$ satisfying the following: There exist $c \in \mathcal{C}$ and $\alpha \in \mathbf{Z}_{+}^{n}$ with $|\alpha| \leq 1$ such that $\Phi^{\alpha}\left(F_{c}^{j 0}, F_{c}^{j 1}, F_{c}^{j 2}\right) \not \equiv 0$.

Set $T(r)=T_{f^{0}}(r)+T_{f^{1}}(r)+T_{f^{2}}(r)$.

We now prove that $\mathcal{Q}=\emptyset$. Suppose that there exists $j_{0} \in \mathcal{Q}$. By Lemma 2 , we have

$$
\begin{aligned}
\| N_{\left(f^{i}, H_{j_{0}}\right)}^{(1)}(r)+4 \sum_{j \in\{1, \ldots, q\} \backslash\left\{j_{0}\right\}} & N_{\left(f^{i}, H_{j}\right)}^{(1)}(r) \\
& \leq N\left(r, \nu_{\Phi^{\alpha}}\right) \leq T(r)+o(T(r)) .
\end{aligned}
$$

$(0 \leq i \leq 2)$.

By Theorem E, we have

$$
\| \sum_{j \neq j_{0}} N_{\left(f^{i}, H_{j}\right)}^{(1)}(r) \geq \frac{q-n-2}{3 n} T(r)+o(T(r))
$$

and

$$
\sum_{j=0}^{q} N_{\left(f^{i}, H_{j}\right)}^{(1)}(r) \geq \frac{q-n-1}{3 n} T(r)+o(T(r)) .
$$

This implies that

$$
\begin{aligned}
\| N_{\left(f^{i}, H_{j_{0}}\right)}^{(1)}(r)+4 \sum_{j \in\{1, \ldots, q\} \backslash\left\{j_{0}\right\}} N_{\left(f^{i}, H_{j}\right)}^{(1)}(r) \\
\quad \geq \frac{4(q-n-2)+1}{3 n} T(r)+o(T(r)) .
\end{aligned}
$$

From (3.12) and (3.13), letting $r \rightarrow \infty$ we get

$$
4(q-n-2)+1 \leq 3 n \Leftrightarrow q \leq \frac{7(n+1)}{4} .
$$

This is a contradiction. Hence $\mathcal{Q}=\emptyset$. Then for each $1 \leq j \leq q, c \in \mathcal{C}$, $\alpha \in \mathbf{Z}_{+}^{n},|\alpha|<2$ we have $\Phi^{\alpha}\left(F_{c}^{j 0}, F_{c}^{j 1}, F_{c}^{j 2}\right) \equiv 0$. Since $\mathcal{C}$ is dense in $\mathbf{C}^{n+1}$, we have that

$$
\Phi^{\alpha}\left(F_{i}^{j 0}, F_{i}^{j 1}, F_{i}^{j 2}\right) \equiv 0(1 \leq i, j \leq q), \text { for all }|\alpha|<2,
$$

where $F_{i}^{j t}:=\frac{\left(f^{t}, H_{j}\right)}{\left(f^{t}, H_{i}\right)}, 0 \leq t \leq 2$. By Lemma $\mathrm{C}$, for each $1 \leq i, j \leq q$, there exists a nonzero constant $\chi_{i j}$ such that $F_{i}^{j 0}=\chi_{i j} F_{i}^{j 1}, F_{i}^{j 1}=\chi_{i j} F_{i}^{j 2}$ or $F_{i}^{j 2}=$ 
$\chi_{i j} F_{i}^{j 0}$. We now show that $\chi_{i j}=1$. Indeed, if $\chi_{i j} \neq 1$, without loss of generality we may assume that $F_{i}^{j 0}=\chi_{i j} F_{i}^{j 1}$. Then $\bigcup_{t \in\{1, \ldots, q\} \backslash\{i, j\}} f^{-1}\left(H_{t}\right)=\emptyset$. Thus, by Theorem E, we have

$$
\|(q-n-3) T_{f}(r) \leq \sum_{t \in\{1, \ldots, q\} \backslash\{i, j\}} N_{\left(f, H_{t}\right)}^{(n)}(r)+o\left(T_{f}(r)\right)=o\left(T_{f}(r)\right) .
$$

Letting $r \longrightarrow+\infty$, we obtain $q-n-3 \leq 0$. This contradicts to $n \geq 2$. Thus,

$$
\chi_{i j}=1 \quad(1 \leq i, j \leq q) .
$$

We take an arbitrary element $k \in\{0,1,2\}$ and an index $i \in\{1, \ldots, q\}$. We will show that $\nu_{\left(f^{k}, H_{i}\right)}=\nu_{\left(f^{l}, H_{i}\right)}$ or $\nu_{\left(f^{k}, H_{i}\right)}=\nu_{\left(f^{t}, H_{i}\right)}$, where $\{l, t\}:=$ $\{0,1,2\} \backslash\{k\}$. In fact, if there is no index $j \neq i$ such that $F_{i}^{j k}=F_{i}^{j l}$ or $F_{i}^{j k}=F_{i}^{j t}$, then since $\chi_{i j}=1$ we have $F_{i}^{j l}=F_{i}^{j t}$ for all $j \neq i$. This implies that $f^{k} \equiv f^{l}$. This is a contradiction. Hence there exists $j \neq i$ such that $F_{i}^{j k}=F_{i}^{j l}$ or $F_{i}^{j k}=F_{i}^{j t}$. This yields that

$$
\nu_{\left(f^{k}, H_{i}\right)}=\nu_{\left(f^{l}, H_{i}\right)} \text { or } \nu_{\left(f^{k}, H_{i}\right)}=\nu_{\left(f^{t}, H_{i}\right)}
$$

for all $k \in\{0,1,2\}, i \in\{1, \ldots, q\}$. For any fixed index $i \in\{1, \ldots, q\}$, by (3.14) (with $k=0$ ) we may assume that $\nu_{\left(f^{0}, H_{i}\right)}=\nu_{\left(f^{1}, H_{i}\right)}$. By (3.14) (with $k=2)$ we obtain $\nu_{\left(f^{2}, H_{i}\right)}=\nu_{\left(f^{0}, H_{i}\right)}$ or $\nu_{\left(f^{2}, H_{i}\right)}=\nu_{\left(f^{1}, H_{i}\right)}$. This implies that $\nu_{\left(f^{0}, H_{i}\right)}=\nu_{\left(f^{1}, H_{i}\right)}=\nu_{\left(f^{2}, H_{i}\right)}$ for all $i \in\{1, \ldots, q\}$. By Lemma 1, we have $f^{0} \equiv f^{1} \equiv f^{2}$. This is a contradiction.

Thus, $\sharp \mathcal{F}\left(f,\left\{H_{i}\right\}_{i=1}^{q}, 2\right) \leq 2$ if

$$
q>\max \left\{\frac{7(N+1)}{4}, \frac{\sqrt{17 N^{2}+16 N}+3 N+4}{4}\right\} .
$$

4. Uniqueness problem for hypersurfaces. Let $f$ be a nonconstant meromorphic mapping of $\mathbf{C}^{m}$ into $\mathbf{C} P^{n}$. We say that a meromorphic function $\varphi$ on $\mathbf{C}^{m}$ is "small" with respect to $f$ if $T_{\varphi}(r)=o\left(T_{f}(r)\right)$ as $r \rightarrow \infty$ (outside a set of finite Lebesgues measure). Denote by $\mathcal{R}_{f}$ the field of all "small" (with respect to $f$ ) meromorphic functions on $\mathbf{C}^{m}$.

Take a reduced representation $\left(f_{0}: \cdots: f_{n}\right)$ of $f$. We say that $f$ is algebraically nondegenerate over $\mathcal{R}_{f}$ if there is no nonzero homogeneous polynomial $Q \in \mathcal{R}_{f}\left[x_{0}, \ldots, x_{n}\right]$ such that $Q(f):=Q\left(f_{0}, \ldots, f_{n}\right) \equiv 0$.

For a homogeneous polynomial $Q \in \mathcal{R}_{f}\left[x_{0}, \ldots, x_{n}\right]$, denote by $Q(z)$ the homogeneous polynomial over $\mathbf{C}$ obtained by substituting a specific point $z \in \mathbf{C}^{m}$ into the coefficients of $Q$.

We say that a set $\left\{Q_{j}\right\}_{j=0}^{n}$ of homogeneous polynomials of the same degree in $\mathcal{R}_{f}\left[x_{0}, \ldots, x_{n}\right]$ is admissible if there exists $z \in \mathbf{C}^{m}$ such that the system 
of equations

$$
\left\{\begin{array}{c}
Q_{j}(z)\left(w_{0}, \ldots, w_{n}\right)=0 \\
0 \leq j \leq n
\end{array}\right.
$$

has only the trivial solution $w=(0, \ldots, 0)$ in $\mathbf{C}^{n+1}$.

First of all, we give the following lemma:

Lemma 3. Let $f$ be a nonconstant meromorphic mapping of $\mathbf{C}^{m}$ into $\mathbf{C} P^{n}$ and $\left\{Q_{j}\right\}_{j=0}^{n}$ be an admissible set of homogeneous polynomials of degree $d$ in $\mathcal{R}_{f}\left[x_{0}, \ldots, x_{n}\right]$. Let $\gamma_{0}, \ldots, \gamma_{n}$ be $(n+1)$ nonzero meromorphic functions in $\mathcal{R}_{f}$.

Put $P=\gamma_{0} Q_{0}^{p}+\cdots+\gamma_{n} Q_{n}^{p}$, where $p$ is a positive integer, $p>n(n+1)$. Assume that $f$ is algebraically nondegenerate over $\mathcal{R}_{f}$. Then

$$
\| d(p-n(n+1)) T_{f}(r) \leq N_{P(f)}^{(n)}(r)+o\left(T_{f}(r)\right) .
$$

Proof. Set $\mathcal{T}_{d}:=\left\{I:=\left(i_{0}, \ldots, i_{n}\right) \in \mathbf{N}_{0}^{n+1}: i_{0}+\cdots+i_{n}=d\right\}$.

Assume that

$$
Q_{j}=\sum_{I \in \mathcal{T}_{d}} a_{j I} x^{I} \quad(j=0, \ldots, n) .
$$

where $a_{j I} \in \mathcal{R}_{f}, x^{I}=x_{0}^{i_{0}} \cdots x_{n}^{i_{n}}$.

Set

$$
F=\left(\gamma_{0} Q_{0}^{p}(f): \cdots: \gamma_{n} Q_{n}^{p}(f)\right): \mathbf{C}^{m} \longrightarrow \mathbf{C} P^{n} .
$$

Since $f$ is algebraically nondegenerate over $\mathcal{R}_{f}$ we have that $F$ is linearly nondegenerate (over $\mathbf{C}$ ).

Assume that $\left(\frac{\gamma_{0} Q_{0}^{p}(f)}{h}: \cdots: \frac{\gamma_{n} Q_{n}^{p}(f)}{h}\right)$ is a reduced representation of $F$, where $h$ is a meromorphic function on $\mathbf{C}^{m}$. Put $F_{i}=\frac{\gamma_{i} Q_{i}^{p}(f)}{h}, i \in\{0, \ldots, n\}$.

We have

$$
\max _{0 \leq j \leq n}\left|Q_{j}^{p}(f)\right| \leq|h| \cdot\left(\sum_{i=0}^{n}\left|\frac{1}{\gamma_{i}}\right|\right) \cdot \max _{1 \leq i \leq n+1}\left|F_{i}\right| .
$$

Let $t=\left(\ldots, t_{k I}, \ldots\right)$ be a family of variables, $\left(k \in\{0, \ldots, n\}, I \in \mathcal{T}_{d}\right)$.

Set

$$
\widetilde{Q}_{j}=\sum_{I \in \mathcal{T}_{d}} t_{j I} x^{I} \in \mathbf{Z}[t, x], \quad j=0, \ldots, n .
$$

Let $\widetilde{R} \in \mathbf{Z}[t]$ be the resultant of $\widetilde{Q}_{0}, \ldots, \widetilde{Q}_{n}$.

Since $\left\{Q_{j}\right\}_{j=0}^{n}$ is an admissible set, $R:=\widetilde{R}\left(\ldots, a_{k I}, \ldots\right) \not \equiv 0$. It is clear that $R \in \mathcal{R}_{f}$ since $a_{k I} \in \mathcal{R}_{f}$.

By Theorems 3.4 and 3.5 in [10], there exists a positive integer $s>d$ and polynomials $\left\{\widetilde{R}_{i j}\right\}_{0 \leq i, j \leq n}$ in $\mathbf{Z}[t, x]$ which are zero or homogeneous in $x$ of 
degree $s-d$ such that

$$
x_{i}^{s} \cdot \widetilde{R}=\sum_{j=0}^{n} \widetilde{R}_{i j} \cdot \widetilde{Q}_{j} \quad \text { for all } i \in\{0, \ldots, n\} .
$$

Set

$$
R_{i j}=\widetilde{R}_{i j}\left(\left(\ldots, a_{k I}, \ldots\right),\left(f_{0}, \ldots, f_{n}\right)\right), \quad 0 \leq i, j \leq n .
$$

Then,

$$
f_{i}^{s} \cdot R=\sum_{j=0}^{n} R_{i j} \cdot Q_{j}\left(f_{0}, \ldots, f_{n}\right) \quad \text { for all } i \in\{0, \ldots, n\}
$$

So,

$$
\begin{aligned}
\left|f_{i}^{s} \cdot R\right| & =\left|\sum_{j=0}^{n} R_{i j} \cdot Q_{j}\left(f_{0}, \ldots, f_{n}\right)\right| \\
& \leq \sum_{j=0}^{n}\left|R_{i j}\right| \cdot \max _{k \in\{0, \ldots, n\}}\left|Q_{k}\left(f_{0}, \ldots, f_{n}\right)\right|
\end{aligned}
$$

for all $i \in\{0, \ldots, n\}$.

We write,

$$
R_{i j}=\sum_{I \in \mathcal{T}_{s-d}} \beta_{I}^{i j} f^{I}, \quad \beta_{I}^{i j} \in \mathcal{R}_{f}
$$

By (4.3), we have

$$
\left|f_{i}^{s} \cdot R\right| \leq\left(\sum_{\substack{0 \leq j \leq n \\ I \in \mathcal{T}_{s-d}}}\left|\beta_{I}^{i j}\right| \cdot\|f\|^{s-d}\right) \cdot \max _{k \in\{0, \ldots, n\}}\left|Q_{k}\left(f_{0}, \ldots, f_{n}\right)\right|,
$$

$i \in\{0, \ldots, n\}$. So,

$$
\frac{\left|f_{i}\right|^{s}}{\|f\|^{s-d}} \leq\left(\sum_{\substack{0 \leq j \leq n \\ I \in \mathcal{T}_{s-d}}}\left|\frac{\beta_{I}^{i j}}{R}\right|\right) \cdot \max _{k \in\{0, \ldots, n\}}\left|Q_{k}\left(f_{0}, \ldots, f_{n}\right)\right|
$$

for all $i \in\{0, \ldots, n\}$.

Thus

$$
\|f\|^{d} \leq\left(\sum_{\substack{0 \leq i, j \leq n \\ I \in \mathcal{T}_{s-d}}}\left|\frac{\beta_{I}^{i j}}{R}\right|\right) \max _{k \in\{0, \ldots, n\}}\left|Q_{k}\left(f_{0}, \ldots, f_{n}\right)\right| .
$$


By (4.1) and (4.4) we have

$$
\|f\|^{d p} \leq\left(\sum_{\substack{0 \leq i, j \leq n \\ I \in \mathcal{T}_{s-d}}}\left|\frac{\beta_{I}^{i j}}{R}\right|\right)^{p} \cdot|h| \cdot\left(\sum_{i=0}^{n}\left|\frac{1}{\gamma_{i}}\right|\right) \cdot\|F\| .
$$

By (4.2) and since $\left(\frac{\gamma_{0} Q_{0}^{p}(f)}{h}: \cdots: \frac{\gamma_{n} Q_{n}^{p}(f)}{h}\right)$ is a reduced representation of $F$, we have

$$
N_{h}(r) \leq p N_{R}(r)+\sum_{i=0}^{n} N_{\gamma_{i}}(r)=o\left(T_{f}(r)\right)
$$

and

$$
N_{\frac{1}{h}}(r) \leq \sum_{\substack{0 \leq j \leq n \\ \bar{I} \in \mathcal{\mathcal { T }}_{d}}} N_{\frac{1}{a_{j I}}}(r)+\sum_{i=0}^{n} N_{\frac{1}{\gamma_{i}}}=o\left(T_{f}(r)\right)
$$

By (4.5), we have

$$
\begin{aligned}
d p \cdot T_{f}(r)= & p d \int_{S(r)} \log \|f\| \sigma+O(1) \\
\leq & \int_{S(r)} \log \left(\sum_{\substack{0 \leq i, j \leq n \\
I \in \mathcal{T}_{s-d}}}\left|\frac{\beta_{I}^{i j}}{R}\right|\right)^{p}|h|\left(\sum_{i=0}^{n}\left|\frac{1}{\gamma_{i}}\right|\right) \sigma+T_{F}(r)+O(1) \\
\leq & p \int_{S(r)} \log ^{+}\left(\sum_{\substack{0 \leq i, j \leq n \\
I \in \mathcal{T}_{s-d}}}\left|\frac{\beta_{I}^{i j}}{R}\right|\right) \sigma+\int_{S(r)} \log ^{+}\left(\sum_{i=0}^{n}\left|\frac{1}{\gamma_{i}}\right|\right) \sigma \\
& +\int_{S(r)} \log |h| \sigma+T_{F}(r)+O(1) \\
\leq & p \sum_{\substack{0 \leq i, j \leq n \\
I \in \mathcal{T}_{s-d}}} m\left(r, \frac{\beta_{I}^{i j}}{R}\right)+\sum_{i=0}^{n} m\left(r, \frac{1}{\gamma_{i}}\right) \\
& +N_{h}(r)-N_{\frac{1}{h}}(r)+T_{F}(r)+O(1) \\
= & T_{F}(r)+o\left(T_{f}(r)\right) .
\end{aligned}
$$


By (4.6) and Theorem E, we have

$$
\begin{aligned}
& \| d p \cdot T_{f}(r) \leq T_{F}(r)+o\left(T_{f}(r)\right) \\
& \leq \sum_{i=0}^{n} N_{\frac{\gamma_{i} Q_{i}^{p}(f)}{h}}^{(n)}(r)+N_{\sum_{i=0}^{n} \frac{\gamma_{i} Q_{i}^{p}(f)}{h}}^{(n)}(r)+o\left(T_{f}(r)\right) \\
& \leq \sum_{i=0}^{n} N_{\frac{\gamma_{i} Q_{i}^{p}(f)}{h}}^{(n)}(r)+N_{\frac{P(f)}{h}}^{(n)}(r)+o\left(T_{f}(r)\right) \\
& \leq \sum_{i=0}^{n} N_{Q_{i}^{p}(f)}^{(n)}(r)+\sum_{i=0}^{n} N_{\gamma_{i}}^{(n)}(r)+(n+2) N_{\frac{1}{h}}(r)+N_{P(f)}^{(n)}(r)+o\left(T_{f}(r)\right) \\
& \leq \sum_{i=0}^{n} n N_{Q_{i}(f)}(r)+N_{P(f)}^{(n)}(r)+o\left(T_{f}(r)\right) \\
& \leq d(n+1) n T_{f}(r)+N_{P(f)}^{(n)}(r)+o\left(T_{f}(r)\right) .
\end{aligned}
$$

This implies that

$$
\| d(p-(n+1) n) T_{f}(r) \leq N_{P(f)}^{(n)}(r)+o\left(T_{f}(r)\right) .
$$

This has completed the proof of the lemma.

Theorem 2. Let $f_{1}, \ldots, f_{k}(k \geq 2)$ be nonconstant meromorphic mappings of $\mathbf{C}^{m}$ into $\mathbf{C} P^{n}$ and $\left\{Q_{j}\right\}_{j=0}^{n}$ be an admissible set of homogeneous polynomials of degree $d$ in $\mathcal{R}_{f_{1}}\left[x_{0}, \ldots, x_{n}\right]$. Let $\gamma_{0}, \ldots, \gamma_{n}$ be $(n+1)$ nonzero meromorphic functions in $\mathcal{R}_{f_{1}}$.

Put $P=\gamma_{0} Q_{0}^{p}+\cdots+\gamma_{n} Q_{n}^{p}$, where $p$ is a positive integer, $p>\frac{n(d(n+1)+k)}{d}$. Assume that $f_{i}$ is algebraically nondegenerate over $\mathcal{R}_{f_{i}}$ for all $i \in\{1, \ldots, k\}$, and

i) $\operatorname{Zero}\left(P\left(f_{i}\right)\right)=\operatorname{Zero}\left(P\left(f_{1}\right)\right)$, for all $i \in\{2, \ldots, k\}$, and

ii) $f_{1} \wedge \cdots \wedge f_{k}=0$ on Zero $\left(P\left(f_{1}\right)\right)$.

Then $f_{1} \wedge \cdots \wedge f_{k} \equiv 0$.

Proof. Assume that $f_{1} \wedge \cdots \wedge f_{k} \not \equiv 0$. We denote by $\mu_{f_{1} \wedge \cdots \wedge f_{k}}$ the divisor associated with $f_{1} \wedge \cdots \wedge f_{k}$. Denote $N_{\mu_{f_{1} \wedge \cdots \wedge f_{k}}}(r)$ the counting function associated with the divisor $\mu_{f_{1} \wedge \cdots \wedge f_{k}}$. It is easy to see that

$$
N_{\mu_{f_{1} \wedge \cdots \wedge f_{k}}}(r) \leq \sum_{i=1}^{k} T_{f_{i}}(r)+O(1) .
$$

Since Zero $\left(P\left(f_{i}\right)\right)=\operatorname{Zero}\left(P\left(f_{1}\right)\right)$, for all $i \in\{2, \ldots, k\}$, we have,

$$
N_{P\left(f_{1}\right)}^{(1)}(r) \leq N_{\mu_{f_{1} \wedge \cdots \wedge f_{k}}}(r) \leq \sum_{i=1}^{k} T_{f_{i}}(r)+O(1) \leq \sum_{i=1}^{k} T_{f_{i}}(r)+O(1) .
$$


Thus, since Zero $\left(P\left(f_{i}\right)\right)=$ Zero $\left(P\left(f_{1}\right)\right)$, for all $i \in\{2, \ldots, k\}$, we have

$$
\sum_{i=1}^{k} N_{P\left(f_{i}\right)}^{(n)}(r) \leq n k N_{P\left(f_{1}\right)}^{(1)}(r) \leq n k \sum_{i=1}^{k} T_{f_{i}}(r)+O(1) .
$$

By Lemma 3 we have

$$
\begin{aligned}
d(p-n(n+1)) T_{f_{1}}(r) & \leq N_{P\left(f_{1}\right)}^{(n)}(r)+o\left(T_{f_{1}}(r)\right) \\
& \leq n N_{P\left(f_{i}\right)}^{(1)}(r)+o\left(T_{f_{1}}(r)\right) \\
& \leq n d p T_{f_{i}}(r)+o\left(T_{f_{1}}(r)\right) \quad(1 \leq i \leq k) .
\end{aligned}
$$

This implies that $\mathcal{R}_{f_{1}} \subset \mathcal{R}_{f_{i}}$ for all $2 \leq i \leq k$. Thus, by Lemma 3 we have

$$
d(p-n(n+1)) T_{f_{i}}(r) \leq N_{P\left(f_{i}\right)}^{(n)}(r)+o\left(T_{f_{i}}(r)\right) \quad(1 \leq i \leq k) .
$$

Combining with (4.7) we have

$$
d(p-n(n+1)) \sum_{i=1}^{k} T_{f_{i}}(r) \leq n k \sum_{i=1}^{k} T_{f_{i}}(r)+o\left(\sum_{i=1}^{k} T_{f_{i}}(r)\right) .
$$

This contradicts to $p>\frac{n(d(n+1)+k)}{d}$. Thus, $f_{1} \wedge \cdots \wedge f_{k} \equiv 0$.

\section{REFERENCES}

[1] Aihara, Y., Finiteness theorem for meromorphic mappings, Osaka J. Math. 35 (1998), 593-61.

[2] Dethloff, G., Tan, T. V., Uniqueness problem for meromorphic mappings with truncated multiplicities and moving targets, Nagoya Math. J. 181 (2006), 75-101.

[3] Dethloff, G., Tan, T. V., Uniqueness problem for meromorphic mappings with truncated multiplicities and few targets, Ann. Fac. Sci. Toulouse Math. (6) 15 (2006), 217-242.

[4] Dethloff, G., Tan, T. V., An extension of uniqueness theorems for meromorphic mappings, Vietnam J. Math. 34 (2006), 71-94.

[5] Fujimoto, H., The uniqueness problem of meromorphic maps into the complex projective space, Nagoya Math. J. 58 (1975), 1-23.

[6] Fujimoto, H., Nonintegrated defect relation for meromorphic maps of complete Kähler manifolds into $\mathbf{P}^{N_{1}}(\mathbf{C}) \times \cdots \times \mathbf{P}^{N_{k}}(\mathbf{C})$, Japan. J. Math. (N. S.) 11 (1985), 233-264.

[7] Fujimoto, H., Uniqueness problem with truncated multiplicities in value distribution theory, Nagoya Math. J. 152 (1998), 131-152.

[8] Fujimoto, H., Uniqueness problem with truncated multiplicities in value distribution theory, II, Nagoya Math. J. 155 (1999), 161-188.

[9] Ji, S., Uniqueness problem without multiplicities in value distribution theory, Pacific J. Math. 135 (1988), 323-348.

[10] Lang, S., Algebra, Third Edition, Addison-Wesley, 1993.

[11] Nevanlinna, R., Einige Eideutigkeitssätze in der Theorie der meromorphen Funktionen, Acta Math. 48 (1926), 367-391.

[12] Noguchi, J., Ochiai, T., Introduction to Geometric Function Theory in Several Complex Variables, Trans. Math. Monogr. 80, Amer. Math. Soc., Providence, Rhode Island, 1990. 
[13] Ru, M., A uniqueness theorem with moving targets without counting multiplicity, Proc. Amer. Math. Soc. 129 (2001), 2701-2707.

[14] Smiley, L., Geometric conditions for unicity of holomorphic curves, Contemp. Math. 25 (1983), 149-154.

[15] Thai, D. D., Quang, S. D., Uniqueness problem with truncated multiplicities of meromorphic mappings in several complex variables, Internat. J. Math. 17 (2006), 12231257.

[16] Thai, D. D., Tan, T. V., Uniqueness problem of meromorphic mappings for moving hypersurfaces, preprint.

[17] Stoll, W., Introduction to value distribution theory of meromorphic maps, Complex analysis (Trieste, 1980), Lecture Notes in Math., 950, Springer, Berlin-New York, 1982, 210-359.

[18] Stoll, W., Value distribution theory for meromorphic maps, Aspects of Mathematics, E 7 Friedr. Vieweg \& Sohn, Braunschweig, 1985.

[19] Stoll, W., On the propagation of dependences, Pacific J. of Math., 139 (1989), 311337.

[20] Ye, Z., A unicity theorem for meromorphic mappings, Houston J. Math. 24 (1998), $519-531$.

Si Duc Quang

Department of Mathematics

Hanoi National University of Education

136-Xuan Thuy street, Cau Giay, Hanoi

Vietnam

e-mail: quangdhsp@yahoo.com

Received February 24, 2008
Tran Van Tan

Department of Mathematics

Hanoi National University of Education

136-Xuan Thuy street, Cau Giay, Hanoi

Vietnam

e-mail: tranvantanhn@yahoo.com 